\title{
The role of quantitative deep capillary plexus in the pathogenesis of type 3 macular neovascularization: an optical coherence tomography angiography study
}

\author{
Marina Concilio $^{1} \cdot$ Federica Fossataro $^{1} \cdot$ Daniela Montorio $^{1} \cdot$ Mariapaola Giordano $^{1} \cdot$ Gilda Cennamo $^{2}$ (I)
}

Received: 23 February 2021 / Revised: 13 June 2021 / Accepted: 17 July 2021 / Published online: 5 August 2021

(c) The Author(s) 2021, corrected publication 2021

\begin{abstract}
Purpose To quantitatively investigate the role of deep capillary plexus (DCP) in patients affected by type 3 macular neovascularization (MNV), compared to patients with reticular pseudodrusen (RPD) eyes and healthy controls, using optical coherence tomography angiography (OCTA).

Methods In this prospective observational study, a total of seventy-eight eyes of 78 patients were included. Group 1 consisted of 40 eyes of 40 patients with stage 1 of type $3 \mathrm{MNV}$ (22 males, 18 females, mean age 73.7, SD \pm 6.60 ) and group 2 included 38 eyes of 38 patients with RPD (17 males, 21 females, mean age 73.2, SD \pm 4.55 ). The control group included 40 eyes of 40 healthy subjects (20 males, 20 females, mean age 71.4, $\mathrm{SD} \pm 6.36$ years). We evaluated the retinal vessel density (VD) of superficial capillary plexus (SCP) and deep capillary plexus (DCP) using OCTA.

Results Patients with diagnosis of type 3 MNV showed statistically lower values of VD in DCP with respect to controls and to RPD group $(p<0.001)$, while there were no statistical differences between RPD and control group in macular region. No significant differences in VD of SCP were detected among the three study groups.

Conclusion OCTA provides a reproducible, non-invasive detailed quantitative analysis of retinal vascular features and changing in early-stage type $3 \mathrm{MNV}$ patients, which allowed to shed the light on the main role of DCP ischemia in the development of type $3 \mathrm{MNV}$.
\end{abstract}

\section{Key messages}

- It is still source of debate the role of retinal and choroidal vascularization in the etiopathogenesis of Type 3 macular neovascularization (MNV)

- Optical coherence tomography angiography (OCTA) may be used as non-invasive tool to provide detailed qualitative and quantitative analysis of the retinal and choriocapillary vascular plexuses

- The analysis of the vessel density of deep capillary plexus (DCP) showed a significant reduction in Type 3 MNV respect to reticular pseudodrusen patients $(p<0.001)$ and to healthy controls $(p<0.001)$

- Quantitative analysis with OCT-A allowed to shed the lights on the role of the impaired deep vascular network in the pathogenesis of Type $3 \mathrm{MNV}$

Keywords Type $3 \mathrm{MNV} \cdot$ Optical coherence tomography angiography $\cdot$ Deep capillary plexus $\cdot$ Vessel density $\cdot$ Reticular pseudodrusen

Gilda Cennamo

xgilda@hotmail.com

Extended author information available on the last page of the article 


\section{Introduction}

Retinal vascular anomalous complex was first described by Hartnett et al. [1] in 1992 as a distinct form of neovascularization (NV) in patients with age-related macular degeneration (AMD). Several hypotheses concerning the origin of this lesion have been postulated. Yannuzzi et al. [2] introduced the term retinal angiomatous proliferation (RAP), proposing an intraretinal origin of the NV. Thus, the authors described an abnormal proliferation of vessels in the middle and inner retina, which spread into the sub-retinal space and eventually communicated with choroidal vessels-the socalled retinal-choroidal anastomosis (RCA) [2]. Conversely, Gass et al. [3] in 2003 hypothesized a choroidal origin and referred to these vascular anomalous complex as occult chorioretinal anastomosis. The term type $3 \mathrm{NV}$, subsequently introduced by Freund et al. [4], expanded the Gass choroidal neovascularization (NVC) classification system omitting its origin.

In the last years, several studies performed to understand the pathogenesis of type $3 \mathrm{MNV}$, supported the intraretinal origin, highlighted early stages' features of type $3 \mathrm{NV}$, and demonstrated the presence of reticular pseudodrusen as a risk factor to the development of type $3 \mathrm{MNV}$ [5-9].

Furthermore, the introduction of optical coherence tomography angiography (OCTA), a novel and non-invasive tool, provided a detailed qualitative and quantitative analysis of the retinal and choroidal vascular plexuses, due to depth-resolved capability, allowing to clarify the type 3 MNV origin.

The aim of this prospective observational study was to quantitatively evaluate deep capillary plexus (DCP) in the early stage of type $3 \mathrm{MNV}$ eyes compared to reticular pseudodrusen (RPD) eyes and healthy controls to better understand the pathophysiologic mechanism of this neovascularization.

\section{Materials and methods}

In this prospective observational study, we recruited seventy-eight eyes of 78 AMD consecutive naïve patients with a diagnosis of type $3 \mathrm{MNV}$ and RPD examined in the Eye Clinic of the University of Naples "Federico II" between September 2019 and December 2020.

The patient group included 40 AMD patients (40 eyes) with stage 1 of type $3 \mathrm{MNV}$, according to Su classification [10], and 38 patients ( 38 eyes) with RPD without drusen and outer retinal atrophy.

The control group consisted of forty eyes of 40 agematched healthy subjects ( 20 males, 20 females, mean age
$71.4, \mathrm{SD} \pm 6.36$ years) with normal ophthalmological examination without ocular and systemic diseases.

Exclusion criteria were previous choroidal neovascularization secondary to other causes, previous anti-VEGF treatments, retinal vascular diseases, myopia greater than 6 diopters, history of ocular surgery, and significant lens opacity.

All the subjects underwent a complete ophthalmological examination including the measurement of best-corrected visual acuity (BCVA), according to the Early Treatment of Diabetic Retinopathy Study (ETDRS), slit-lamp biomicroscopy, fundus examination, multimodal imaging, namely multicolor imaging, infra-red (IR), fundus autofluorescence (FAF), fluorescein angiography (FA), indocyanine green angiography (ICGA), spectral-domain (SD)-optical coherence tomography (OCT) (Spectralis, Heidelberg Engineering, Heidelberg, Germany), and OCTA (AngioVue, RTVue XR Avanti, Optovue, Inc., Freemont, CA). All observations and the OCTA measurements were performed by two masked examiners (MC, DM) and a senior expert (GC) that confirmed the diagnosis of stage 1 type $3 \mathrm{MNV}$.

The study was approved by the Institutional Review Board of the University of Naples "Federico II" and all investigations adhered to the tenets of the Declaration of Helsinki. Written informed consents were obtained from the patients enrolled in the study. The research protocol was registered on clinical Trials.gov (protocol number: 2912/20).

\section{Optical coherence tomography angiography}

OCTA images with the Optovue Angiovue System (software ReVue XR version 2018.1.1.60, Optovue Inc., Fremont, CA, USA) followed a standardized protocol based on the splitspectrum amplitude-decorrelation algorithm (SSADA), as previously described [11].

A $6 \mathrm{~mm} \times 6 \mathrm{~mm}$ macular scan was performed to visualize the retinal capillary plexus in the macular region divided, according to the ETDRS classification of diabetic retinopathy, in the whole image, fovea, and parafovea.

The AngioAnalytic ${ }^{\mathrm{TM}}$ software automatically calculated in the two retinal vascular networks: superficial capillary plexus (SCP) and DCP, the vessel density (VD), defined as the percentage area occupied by the microvasculature in whole scan area and in all sections [12].

The software includes the 3D Projection Artifact Removal algorithm to improve the quality of OCTA images.

From the analysis were excluded the images with a signal strength index less than 60 and residual motion artefacts, incorrect segmentation, low centration, and focus.

\section{Statistical analysis}

Statistical analysis was performed with the Statistical Package for Social Sciences (Version 25 for Windows; SPSS Inc, 
Chicago, IL, USA). One-way analysis of variance (ANOVA) followed by Bonferroni post hoc analysis was used to compare OCTA parameters among controls, type $3 \mathrm{MNV}$, and RPD groups. A $p$ value of $<0.05$ was considered statistically significant.

\section{Results}

A total of seventy-eight eyes of 78 patients including 40 AMD patients with type $3 \mathrm{MNV}$ (22 males, 18 females, mean age 73.7, $\mathrm{SD} \pm 6.60)$ and $38 \mathrm{RPD}$ patients (17 males, 21 females, mean age $73.2, \mathrm{SD} \pm 4.55)$ was enrolled in this study.

The age and sex did not significantly differ among the study groups ( $p=0.191, p=0.663$; respectively).

BCVA was significantly impaired in type $3 \mathrm{MNV}$ patients with respect to other groups $(p<0.001)$ while it did not differ between RPD patients and controls (Table 1).

OCTA examination showed no statistically significant difference in VD of SCP among the three study groups in the macular region.

Different results were found in DPC analysis that revealed statistically lower values of VD in type $3 \mathrm{MNV}$ patients with respect to controls $(p<0.001)$ while there were no statistical differences between the RPD group and controls in each macular sector (Fig. 1).

Comparing the two study groups, the VD of DCP was significantly decreased in type $3 \mathrm{MNV}$ with respect to RPD patients (whole image $p<0.001$; parafovea $p<0.001$; fovea $p<0.001$ ) (Table 2).

\section{Discussion}

To the best of our knowledge, no previous studies have been published about the quantitative analysis of DCP in the early stage of type $3 \mathrm{MNV}$, with OCTA. However, several reports analyzed in detail the microvascular morphology of type 3 MNV supporting the origin from the DCP and reporting the choroidal anomalies associated with this type of NV using OCT and OCTA [13-16].

A distinct tuft-like network, with high flow, origins at the DCP which could later progress towards the sub-retinal pigment epithelium (RPE) space, the RPE, and the choroid, causing exudation and pigment epithelial detachment, was described $[10,17,18]$.

Consequently, different plexuses could get involved during type $3 \mathrm{MNV}$ progression.

Sacconi et al. [5] first described the characteristics of early intraretinal $\mathrm{NV}$ originating from $\mathrm{DCP}$, as the presence of intraretinal hyperreflective foci on structural OCT, corresponding to a detectable flow in DCP on OCTA in nascent type $3 \mathrm{NV}$. In both the preclinical stage and stage 1, the retinal vascular proliferation does not show a visible connection to the RPE and sub-RPE space or the choroid [5, 10]. Therefore, in the early stages of type $3 \mathrm{MNV}$, the DCP could be the first plexus involved.

In our prospective observational study, we evaluated VD of DCP in type $3 \mathrm{MNV}$ eyes with stage 1 versus RPD eyes and healthy controls, using OCTA, to better understand the role of DPC in the pathogenesis of this type of NV.

Overall, we found a lower perfusion of the inner retina in the type $3 \mathrm{MNV}$ group compared with RPD patients, while a mild decrease of VD, although not statically significant, was found in the RPD group versus controls.

Martins et al. [6] also noticed a significantly lower vascular perfusion of the DCP in patients with RAP.

Furthermore, in support of a retinal origin of type $3 \mathrm{MNV}$, several histopathologic studies demonstrated the evidence of intraretinal NV without the presence of sub-RPE vascular involvement $[19,20]$. Therefore, it is plausible to hypothesize that an alteration of the DCP, specifically a reduction in $\mathrm{VD}$, may favor the subsequent development of type $3 \mathrm{MNV}$. Retinal ischemia, due to a reduction in blood flow, with the
Table 1 Demographic, ophthalmological characteristics of controls, RPD, and type 3 MNV patients

\begin{tabular}{lllll}
\hline & Control group & RPD & Type 3 MNV & $\begin{array}{l}\text { ANOVA } \\
p\end{array}$ \\
\hline Eyes (n.) & 40 & 38 & 40 & - \\
Gender (male/female) & $20 / 20$ & $17 / 21$ & $22 / 18$ & $0.663 \dagger$ \\
Age (years) & $71.4 \pm 6.36$ & $73.2 \pm 4.55$ & $73.7 \pm 6.60$ & 0.191 \\
BCVA (LogMAR) & $0.16 \pm 0.07$ & $0.19 \pm 0.07$ & $0.35 \pm 0.07$ & $<0.001$ \\
\hline
\end{tabular}

Data are expressed as mean $\pm \mathrm{SD}$

$R P D$ reticular pseudodrusen; $M N V$ macular neovascularization

$B C V A$ best-corrected visual acuity; $\log M A R \operatorname{logarithm}$ of the minimum angle of resolution

One-way analysis of variance (ANOVA) followed by Bonferroni post hoc analysis

${ }^{\dagger}$ Chi-squared test, statistical significance $P$ value $<0.05$

Statistical significance $P$ value $<0.05$ 

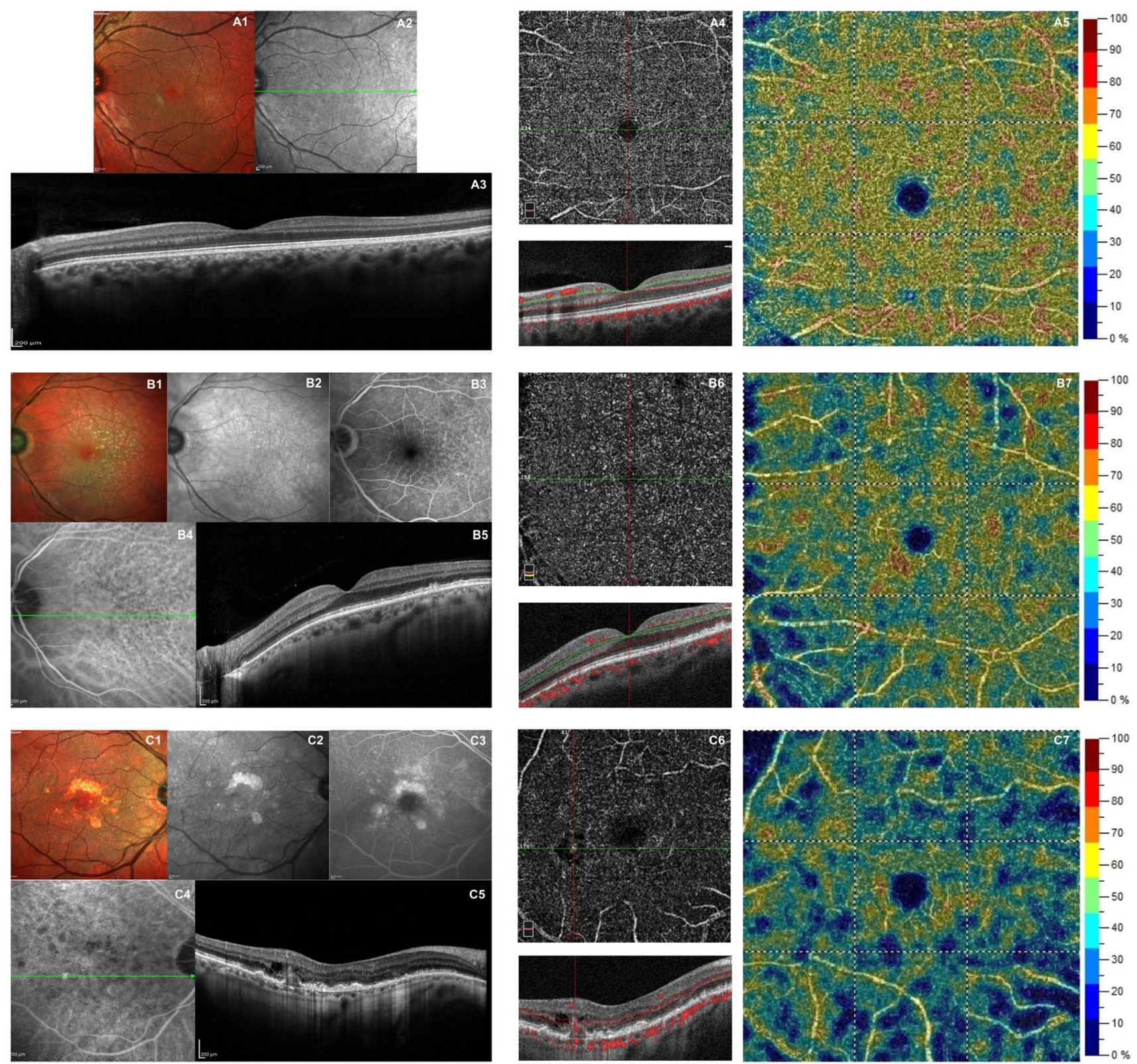

Fig. 1 Top row. Left eye of a healthy subject (68-year-old female) shows a normal multicolor (A1) and infra-red imaging (A2), absence of structural retinal changes at spectral-domain optical coherence tomography (SD-OCT) B-scan (A3), normal morphology of deep capillary plexus (DCP) (A4), and normal vessel density of the DCP (A5) at OCT angiography (OCTA). Middle row. Left eye of a patient (65-year-old male) with reticular pseudodrusen reveals a yellowishgreen reticular pattern at multicolor imaging (B1), doughnut pattern at infra-red imaging (B2), hyperreflective points at fluorescein angiography (B3), hyporeflective circular pattern at indocyanine green angiography (B4), and multiple accumulation of hyperreflective material above the retinal pigment epithelium (B5) at structural SDOCT B-scan. OCTA images of the DCP (B6) present a focal reduction of vessel density in DCP (B7) respect to healthy subject. Bottom row. Right eye of a patient (64-year-old female) with diagnosis of type $3 \mathrm{MNV}$ in stage 1 reveals yellowish-green reticular pattern, large yellowish drusen, and hemorrhage in macular region at multicolor imaging (C1), hyperreflective pattern and hyporeflective point corresponding to the hemorrhage at infra-red imaging (C2), and an area of hyperfluorescence at fluorescein angiography (C3). Indocyanine green angiography shows a focal area of hyperfluorescence or so-called "hot spot" representing the type 3 neovascularization (C4). SD-OCT shows corresponding reflectance within the retina at the level of outer and inner nuclear layers overlying a reticular pseudodrusen, with development of intraretinal fluid (C5). OCTA images reveals a tuft-like network with dark halo at the level of DCP with corresponding flow signal at the structural OCT B-scan (C6). A widespread reduction of vessel density of DCP was detected at OCTA respect to eyes with reticular pseudodrusen and healthy subjects $(\mathrm{C} 7)$ 
Table 2 Comparison of retinal vessel density among controls, RPD, and type $3 \mathrm{MNV}$ patients

\begin{tabular}{|c|c|c|c|c|c|c|c|}
\hline & Control group & RPD & Control vs RPD & Type $3 \mathrm{MNV}$ & $\begin{array}{l}\text { Control vs type } \\
3 \mathrm{MNV}\end{array}$ & $\begin{array}{l}\text { RPD vs type } 3 \\
\text { MNV }\end{array}$ & $\begin{array}{l}\text { ANOVA } \\
p\end{array}$ \\
\hline \multicolumn{8}{|l|}{$\mathrm{SCP}(\%)$} \\
\hline Whole image & $46.76 \pm 8.32$ & $43.98 \pm 7.18$ & 0.375 & $42.90 \pm 8.16$ & 0.094 & 0.852 & 0.085 \\
\hline Parafovea & $47.87 \pm 7.07$ & $45.85 \pm 8.57$ & 0.687 & $45.28 \pm 6.29$ & 0.354 & 1 & 0.260 \\
\hline Fovea & $29.90 \pm 6.65$ & $27.91 \pm 6.97$ & 0.697 & $26.53 \pm 8.15$ & 0.123 & 0.921 & 0.120 \\
\hline \multicolumn{8}{|l|}{$\mathrm{DCP}(\%)$} \\
\hline Whole image & $49.32 \pm 7.43$ & $46.91 \pm 7.92$ & 0.426 & $42.12 \pm 6.17$ & $<0.001$ & 0.012 & $<0.001$ \\
\hline Parafovea & $51.31 \pm 7.34$ & $48.12 \pm 8.59$ & 0.222 & $43.58 \pm 7.49$ & $<0.001$ & 0.035 & $<0.001$ \\
\hline Fovea & $41.64 \pm 7.39$ & $38.24 \pm 7.24$ & 0.170 & $33.28 \pm 8.61$ & $<0.001$ & 0.017 & $<0.001$ \\
\hline
\end{tabular}

Data are expressed as mean $\pm \mathrm{SD}$

$R P D$ reticular pseudodrusen; $M N V$ macular neovascularization

$S C P$ superficial capillary plexus; $D C P$ deep capillary plexus

One-way analysis of variance (ANOVA) followed by Bonferroni post hoc analysis

Statistical significance $P$ value $<0.05$

consequent increase in VEGF could represent the primum movens of hypoxia [21].

Moreover, the changes of DCP were highlighted by Colantuono et al. [22] who suggested that, in different agerelated degeneration (AMD) stages, alterations in both outer retina and RPE, which represent the main metabolic consumer in the retina, may cause an increase of oxygen from the choroid to the inner retina and, therefore, a vasoconstriction, visualized with a decrease of retinal perfusion in DCP on OCTA. The authors, indeed found a reduction of DCP in eyes with quiescent macular NV and intermediate AMD, while they report that in exudative AMD eyes, the perfusion density remained stable throughout follow-up, suggesting that anti-vascular endothelial growth factor (VEGF) injections do not impact on the perfusion of the DCP [22].

On the other hand, Borrelli et al. [23] showed that morphology of retinal capillary plexus is strongly linked to the metabolic demand of neuroretina and to its thickness, especially for DCP perfusion, demonstrating a direct relation between these two variables.

Therefore, a reduced perfusion of the inner retina could suggest that the early changes in DCP might represent a possible biomarker of type $3 \mathrm{MNV}$ formation and our results could hence confirm the primary role of the DCP in the pathogenesis of type $3 \mathrm{MNV}$.

Furthermore, we also found a mild decrease in VD of DCP, although not statically significant, in the RPD group versus controls. Moreover, previous studies showed a reduction of choriocapillaris VD in RPD, as well as in eyes with type $3 \mathrm{MNV}$ suggesting that this hypoperfusion could represent a hypoxic precursor for the onset of this MNV [13, 24, 25]. Therefore, the reduction of retinal and choriocapillaris VD may suggest a common origin of type $3 \mathrm{MNV}$ and RPD and raise further interesting questions about the possible role of RPD as the precursor of type $3 \mathrm{MNV}$.
Future longitudinal studies on larger cohorts may more precisely evaluate the variations of DCP in the different stages of type $3 \mathrm{MNV}$, its causative role in the development and progression of this type of $\mathrm{NV}$, and the possible correlation with the role of both choriocapillaris and choroid. This study had several limitations: firstly, the small sample size. Secondly, one of the most notable limitations of OCTA is image artefacts, due to distorted retinal structures and projection effects, which more profoundly impact on DCP evaluation rather than SCP and which we overcome using projection-resolved software.

In conclusion, our finding could shed the light on the role of DCP in the pathogenesis of type $3 \mathrm{MNV}$ due to a detailed quantitative analysis of retinal vascular features provided by OCTA.

Funding Open access funding provided by Università degli Studi di Napoli Federico IIwithin the CRUI-CARE Agreement.

\section{Declarations}

Ethical approval All procedures performed in studies involving human participants were in accordance with the ethical standards of the Institutional Review Board of the University of Naples "Federico II" and with the 1964 Helsinki Declaration and its later amendments or comparable ethical standards.

Informed consent Informed consent was obtained from all individual participants included in the study.

Conflict of interest The authors declare no competing interests.

Open Access This article is licensed under a Creative Commons Attribution 4.0 International License, which permits use, sharing, adaptation, distribution and reproduction in any medium or format, as long as you give appropriate credit to the original author(s) and the source, provide a link to the Creative Commons licence, and indicate if changes were made. The images or other third party material in this article are 
included in the article's Creative Commons licence, unless indicated otherwise in a credit line to the material. If material is not included in the article's Creative Commons licence and your intended use is not permitted by statutory regulation or exceeds the permitted use, you will need to obtain permission directly from the copyright holder. To view a copy of this licence, visit http://creativecommons.org/licenses/by/4.0/.

\section{References}

1. Hartnett ME, Weiter JJ, Garsd A, Jalkh AE (1992) Classification of retinal pigment epithelial detachments associated with drusen. Graefes Arch Clin Exp Ophthalmol 230:11-19

2. Yannuzzi LA, Negrao S, Iida T et al (2001) Retinal angiomatous proliferation in age-related macular degeneration. Retina 21:416-434

3. Gass JD, Agarwal A, Lavina AM, Tawansy KA (2003) Focal inner retinal hemorrahages in patients with drusen: an early sign of occult choroidal neovascularization and chorioretinal anastomosis. Retina 23:741-751

4. Freund KB, Ho IV, Barbazetto IA et al (2008) Type 3 neovascularization: the expanded spectrum of retinal angiomatous proliferation. Retina 28(2):201-11

5. Sacconi R, Sarraf D, Garrity S et al (2018) Nascent type 3 neovascularization in age-related macular degeneration. Ophthalmol Retina 2(11):1097-1106

6. Martins A, Farinha C, Raimundo M et al (2018) Multimodal evaluation of the fellow eye of patietns with retinal angiomatous proliferation. Ophthalmic Res 59(2):88-97

7. Savastano MC, Lumbroso B, Rispoli M (2015) In vivo characterization of retinal vascularization morphology using optical coherence tomography angiography. Retina 35:2196-2203

8. Spaide RF (2016) Choriocapillaris flow features flow a power law distribution: implications for characterization and mechanisms of disease profession. Am J Ophthalmol 170:58-67

9. Sawa M, Ueno C, Gomi F, Nishida K (2014) Incidence and characteristics of neovascularization in fellow eyes of Japanese patients with unilateral retinal angiomatous proliferation. Retina 34:761-767

10. Su D, Lin S, Phasukkijwatana N et al (2016) An updated staging system of type 3 neovascularization using spectral domain optical coherence tomography. Retina 36(1):S40-S49

11. Jia Y, Tan O, Tokayer J et al (2012) Split spectrum amplitudedecorrelation angiography with optical coherence tomography. Opt Express 20:4710-4725

12. Huang D, Jia Y, Gao SS, Lumbroso B, Rispoli M (2016) Optical coherence tomography angiography using the Optovue device. Dev Ophthalmol 56:6-12
13. Borrelli E, Souied EH, Freund KB (2018) Reduced choriocapillaris flow in eyes with type 3 neovascularization and age-related macular degeneration. Retina 38:1968-1976

14. Phasukkijwatana N, Tan ACS, Chen X et al (2017) Optical coherence tomography angiography of type 3 neovascularization in agerelated macular degeneration after antiangiogenic therapy. $\mathrm{Br} \mathbf{J}$ Ophthalmol 101:597-602

15. Querques G, Miere A, Souied EH (2016) Optical coherence tomography angiography features of type 3 neovascularization in age-related macular degeneration. Dev Ophthalmol 56:57-61

16. Sacconi R, Battista M, Borrelli E et al (2021) OCT-A characterisation of recurrent type 3 macular neovascularisation. Br J Ophthalmol 105:222-226

17. Nagiel A, Sarraf D, Sadda SR et al (2015) Type 3 neovascularization evolution, association with pigment epithelial detachment, and treatment response as revealed by spectral domain optical coherence tomography. Retina 35:638-647

18. Silva N, Marta A, Baptista P, Furtado MJ, Lume M (2020) Optical coherence tomography findings (SD-OCT and OCTA) in early-stage type 3 neovascularization. Case Rep Ophthalmol 11:493-499

19. Lafaut BA, Aisenbrey S, Broecke CV, Bartz-Schmidt KU (2000) Clinicopathological correlation of deep retinal vascular anomalous complex in age-related macular degeneration. Br J Ophthalmol 84:1269-1274

20. Monson DM, Smith JR, Klein ML, Wilson DJ (2008) Clinicopathologic correlation of retinal angiomatous proliferation. Arch Ophthalmol 126:1664-1668

21. Feigl B (2009) Age-related maculopathy - linking aetiology and pathophysiological changes to the ischaemia hypothesis. Prog Retin Eye Res 28(1):63-86

22. Colantuono D, Souied EH, Borrelli E et al (2020) Quantitative deep vascular complex analysis of different AMD stages on optical coherence tomography angiography. Eur J Ophthamol 3;1120672120968758

23. Borrelli E, Toto L, Viggiano P et al (2020) Widefield topographical analysis of the retinal perfusion and neuroretinal thickness in healthy eyes: a pilot study. Eye 34(12):2264-2270

24. Montorio D, D'andrea L, Mirto N, Cennamo G (2020) The role of optical coherence tomography angiography in reticular pseudodrusen. Photodiagnosis Protodyn Ther 33:102094

25. Mai LEH, Souied EH, Querques G et al (2021) Choriocapillaris flow impairment in type 3 macular neovascularization: a quantitative analysis using swept-source optical coherence tomography angiography. Retina 12

Publisher's note Springer Nature remains neutral with regard to jurisdictional claims in published maps and institutional affiliations.

\section{Authors and Affiliations}

\section{Marina Concilio $^{1} \cdot$ Federica Fossataro $^{1} \cdot$ Daniela Montorio $^{1} \cdot$ Mariapaola Giordano $^{1} \cdot$ Gilda Cennamo $^{2}$ (i)}

1 Eye Clinic, Department of Neurosciences, Reproductive Sciences and Dentistry, University of Naples Federico II, Via S. Pansini 5, 80131 Naples, Italy
2 Eye Clinic, Department of Public Health, Federico II University, Naples, Italy 